\title{
Proceeding Paper \\ Mitigation of Circulating Currents for Parallel Connected Sources in a Standalone DC Microgrid ${ }^{\dagger}$
}

\author{
Muhammad Rashad ${ }^{1, *}$, Uzair Raoof ${ }^{1}$, Nazam Siddique ${ }^{2}$ and Daud Mustafa Minhas ${ }^{3}$ (D) \\ 1 Department of Electrical Engineering, The University of Lahore (UOL), Lahore 54000, Pakistan; \\ uzair@uol.edu.pk \\ 2 Department of Electrical Engineering, University of Gujrat (UOG), Gujrat 50700, Pakistan \\ nazim.siddiq@gmail.com \\ 3 Department of Systems Engineering, Saarland University, D-66123 Saarbrucken, Germany; \\ daud.minhas@aut.uni-saarland.de \\ * Correspondence: muhammad.rashad@ee.uol.edu.pk \\ $\dagger$ Presented at the 1st International Conference on Energy, Power and Environment, Gujrat, Pakistan, \\ 11-12 November 2021.
}

Citation: Rashad, M.; Raoof, U.;

Siddique, N.; Minhas, D.M.

Mitigation of Circulating Currents for

Parallel Connected Sources in a Standalone DC Microgrid. Eng. Proc. 2021, 12, 31. https://doi.org/ 10.3390/engproc2021012031

Academic Editor: Shahid Iqbal

Published: 24 December 2021

Publisher's Note: MDPI stays neutral with regard to jurisdictional claims in published maps and institutional affiliations.

Copyright: (C) 2021 by the authors. Licensee MDPI, Basel, Switzerland. This article is an open access article distributed under the terms and conditions of the Creative Commons Attribution (CC BY) license (https:// creativecommons.org/licenses/by/ $4.0 /)$.

\begin{abstract}
In a standalone DC microgrid, sources are interconnected in a parallel configuration. When sources of different power ratings are parallel connected, there arises a major issue of circulating currents which disturb current sharing by sources as per their capacity. Consequently, the voltage regulation becomes poorer. Additionally, connecting line resistances also play their part to contribute to abnormal current sharing. Droop controllers are normally preferred for the mitigation of circulating currents among parallel-connected sources. However, droop controllers cannot eliminate circulating currents for different rating sources. Hence, current sharing and voltage regulation cannot be ensured simultaneously. To address the issues, a distributed architecture-based Sliding Mode Control (SMC) technique is proposed in this paper. An analysis of the circulating currents for a two-source system is presented. Simulation results are presented to show the effectiveness and fail-safe operation of the proposed technique in a steady-state condition.
\end{abstract}

Keywords: microgrid; circulating current; voltage regulation; droop controller; sliding mode control

\section{Introduction}

Microgrids are the modern form of self-controlled grids that can efficiently generate, store and consume electric power. Thus, two types of Power Electronic Converters (PECs) are required to integrate RESs and loads with the AC line, whereas in DC microgrids, the line is DC. The required number of PECs in DC microgrids is less and hence conversion losses are reduced [1]. Furthermore, skin effect, frequency synchronization, and power factor problems are absent in DC microgrids which makes them attractive for efficiently generate and utilize power [2].

Sources through PECs are connected in a parallel configuration in DC microgrids sharing a common DC line as shown in Figure $1[3,4]$. Minimizing circulating currents is the main control objective in a DC microgrid, which will improve load sharing among sources and voltage regulation. To address the problem, various control architectures have been proposed in literature which can be divided into centralized and decentralized control. Centralized architecture can achieve the objective utilizing a high-bandwidth communication link, but a single point failure can degrade the performance and reliability $[5,6]$. In decentralized architecture, the microgrid lacks the information of the parallel-connected sources which cannot achieve the current sharing and voltage regulation simultaneously [7,8]. To address the issue, distributed control architecture utilizing low bandwidth communication is proposed in this paper. 


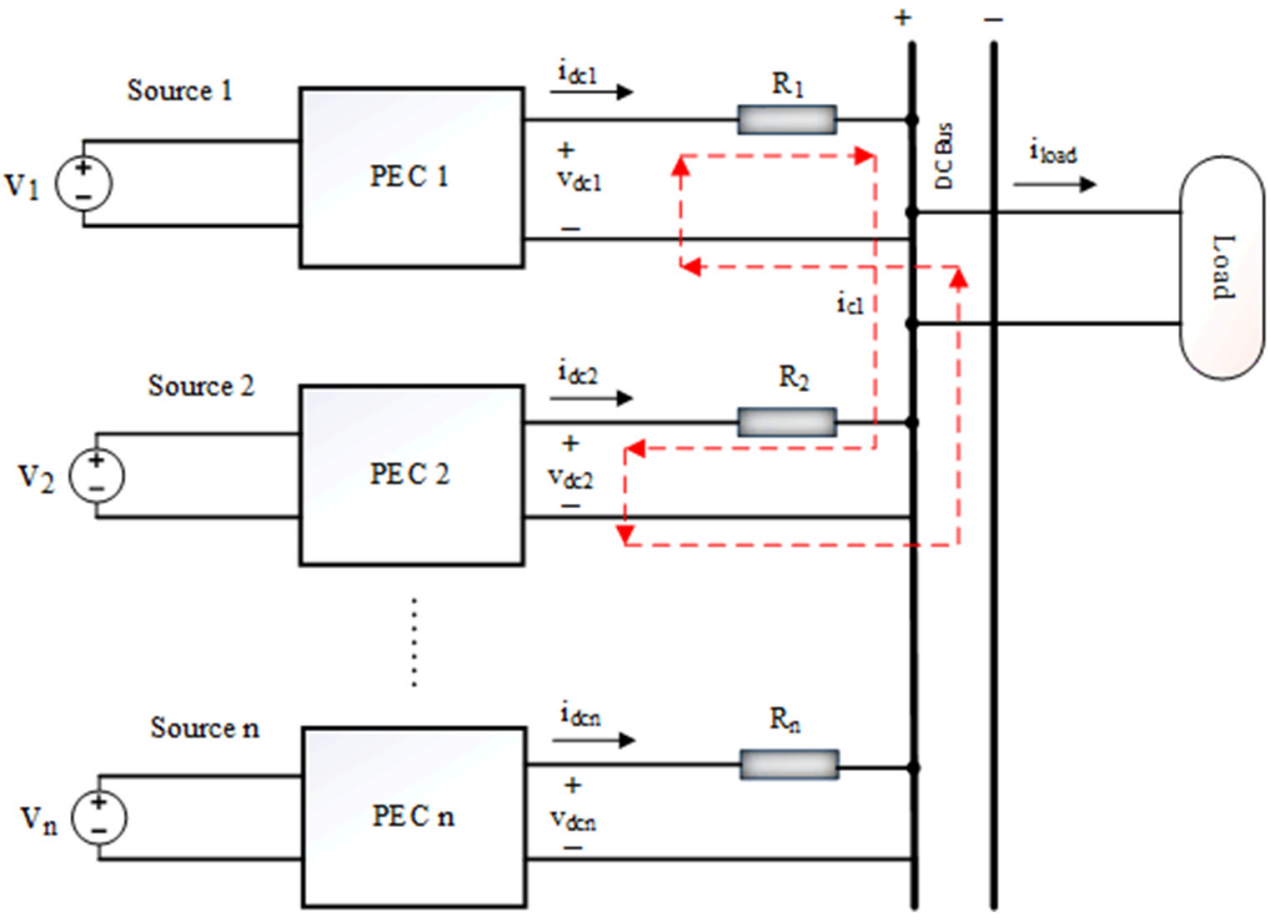

Figure 1. DC microgrid configuration.

Droop-based PI control techniques are normally preferred for inner voltage and current loops of the PECs of parallel-connected sources. PI controllers lack the global stability of the system. Additionally, it is difficult to optimize the control parameters with the change in operating conditions of the system [9]. Therefore, droop controllers are not recommended for eliminating circulating currents among parallel-connected sources. Alternatively, a Sliding Mode Control (SMC) technique is proposed in this paper. It is a nonlinear-type controller which ensures stability in all operating conditions [10]. Therefore, SMC is proposed to control the inner voltage and current loop of the PECs.

The organization of the paper is as follows. Section 2 deals with the analysis of circulating currents. Section 3 presents the droop control and Section 4 presents the proposed distributed architecture based on SMC for eliminating the circulating currents. The results are presented in Sections 5 and 6 concludes this paper.

\section{Circulating Current Analysis}

To analyze the circulating currents, consider a DC microgrid in which two sources are connected in parallel configuration sharing a common load as shown in Figure 1. The problem of circulating currents can arise due to differences in the connecting line resistances and output voltages. These contribute to losses and affect the load sharing among the sources. A Thevenin equivalent circuit is shown in Figure 2. The following equations can be written applying Kirchoff's Voltage Law (KVL) in Figure 3.

$$
\begin{gathered}
v_{d c 1}=i_{d c 1} R_{1}+i_{L} R_{L} \\
v_{d c 2}=i_{d c 2} R_{2}+i_{L} R_{L} \\
i_{L}=i_{L 1}+i_{L 2} \\
i_{d c 1}=i_{L 1}+i_{c 1} \\
i_{d c 2}=i_{L 2}+i_{c 2}
\end{gathered}
$$

where $v_{d c 1}$, and $v_{d c 2}$, are the voltage of PEC 1 and $2, i_{d c 1}$, and $i_{d c 2}$, are the currents supplied by source 1 and $2 R_{1}$, and $R_{2}$ are line resistances, $R_{L}$, and $i_{L}$ are load resistance and 
current, respectively. $i_{L 1}$ and $i_{L 2}$ are the contribution to load current by source 1 and 2; $i_{c 1}$ and $i_{c 2}$ are circulating currents, respectively.

Node-1

Node- 2

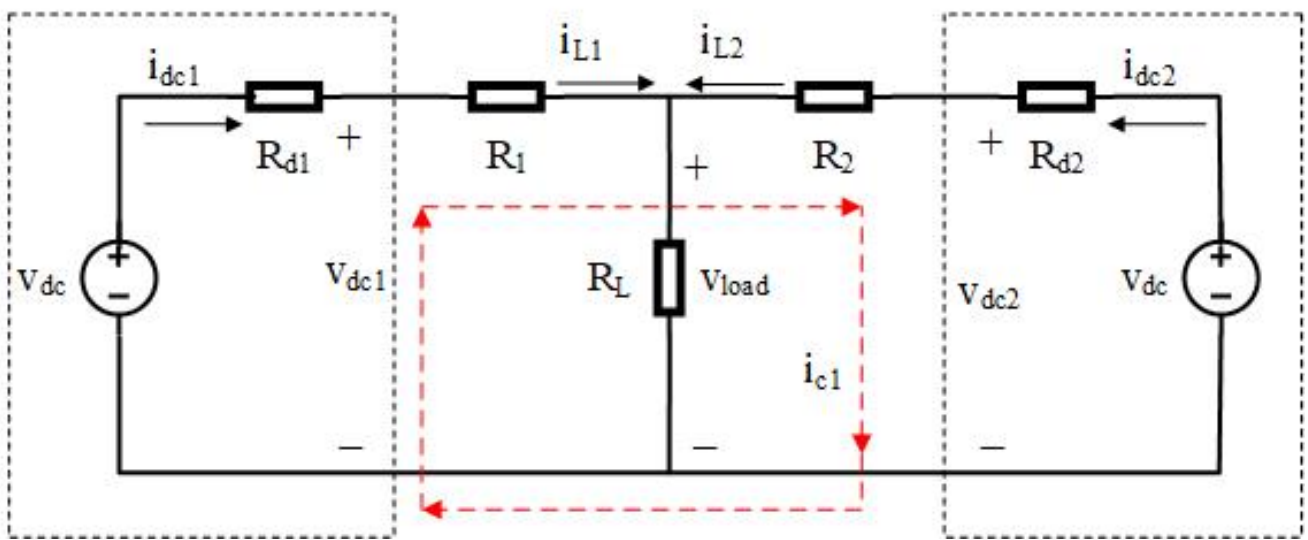

Figure 2. DC microgrid configuration.

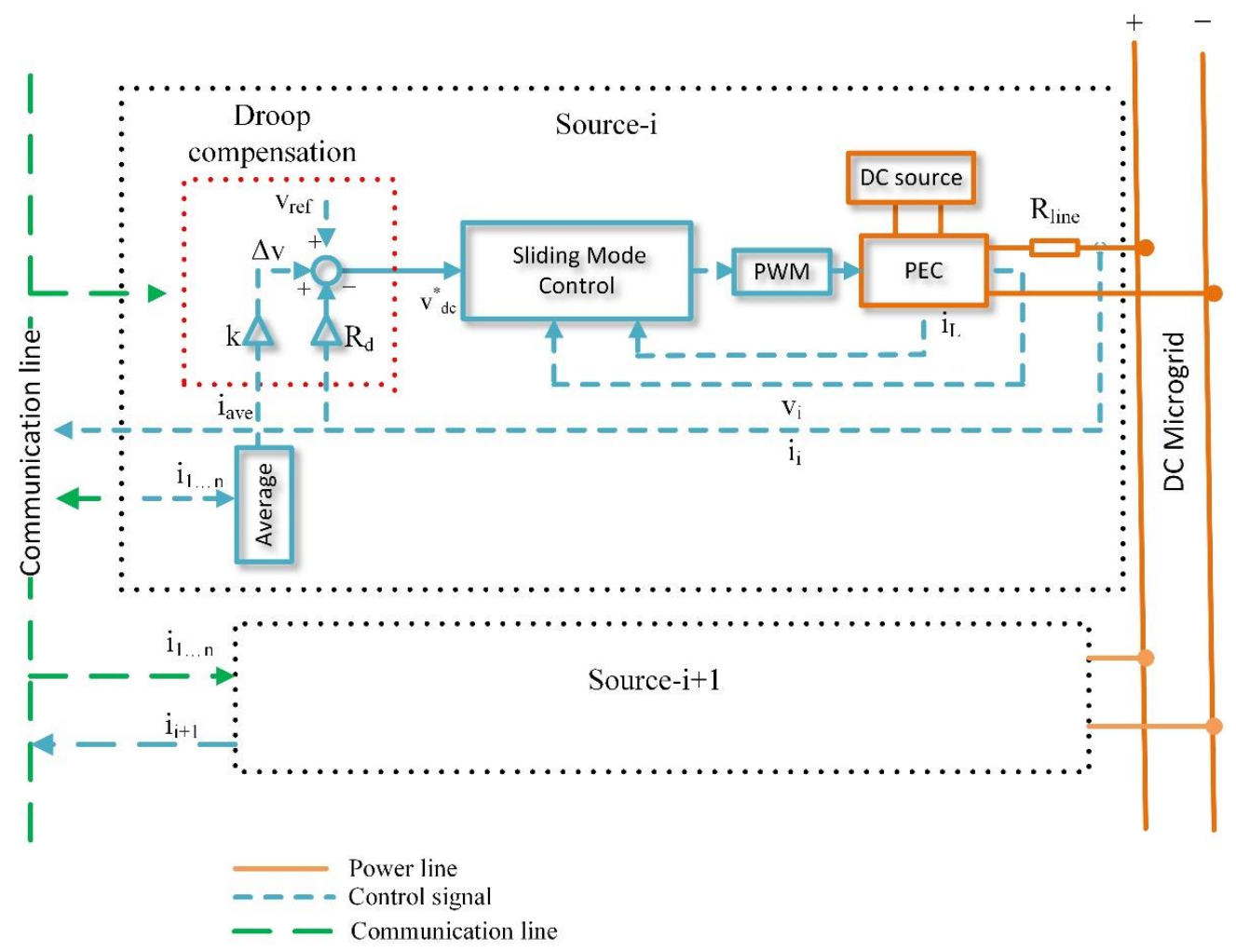

Figure 3. Distributed control architecture.

The circulating currents $i_{c 1}$ and $i_{c 2}$ are equal in magnitude but opposite in direction, and can be written after simplification as:

$$
i_{c 1}=-i_{c 2}=\frac{v_{d c 1}-v_{d c 2}}{R_{1}+R_{2}}=\frac{R_{1} i_{d c 1}-R_{2} i_{d c 2}}{R_{1}+R_{2}} \quad\left(R_{1} \neq R_{2}\right)=\frac{i_{d c 1}-i_{d c 2}}{2} \quad\left(R_{1}=R_{2}\right)
$$

The Equation (6) shows the contributed value of the circulating current in the supplied current by sources 1 and 2 . 


\section{Droop Control}

In droop control, current sharing among paralleled sources is increased by adding series resistance in the path of the source current. The relationship commonly defined by the droop action is given as:

$$
v_{d c}^{*}=v_{r e f}-i_{d c i} R_{d i}
$$

where $v_{d c^{\prime}}^{*} v_{r e f}$ and $R_{d i}$ are the output voltage, reference voltage and droop resistance, respectively. The maximum value of the droop resistance is selected based on the condition given as [8]:

$$
R_{d i} \leq \frac{\Delta v_{\max }}{i_{\text {dci_max }}}
$$

where $\Delta v_{\max }$ is the maximum deviation that can be allowed and $i_{d c i_{-} \text {max }}$ is the maximum source current.

\section{Proposed Control Architecture}

Distributed control architecture, which is a substitute to the single central controller, is proposed to eliminate the circulating currents for parallel connected sources in a DC microgrid. The proposed architecture is shown in Figure 3. The large value of the droop resistance $R_{d}$ can mitigate the circulating currents but voltage regulation is disturbed. To address the issue, a modified droop action is defined as:

$$
v_{d c}^{*}=v_{r e f}+\Delta v_{i}-i_{d c i} R_{d i}
$$

where $\Delta v_{i}$ is the value of voltage shift, which is to be increased as the load current increases so that the deviation due to the factor $i_{d c i} R_{d i}$ can be compensated. To find out the compensated value of $\Delta v_{i}$, the supplied current of each source is shared with the other sources using Controller Area Network (CAN), a low-bandwidth communication, as shown in Figure 3. The distributive controller determines the average value of the supplied current through the relationship given as:

$$
i_{\text {avg }}=\frac{\sum_{m=1}^{n} i_{m}}{n}
$$

where $n$ is the number of sources in parallel configuration and $i_{m}$ is the current supplied by the $m$ th source, respectively. The value of $\Delta v_{i}$ is calculated through the relationship given as:

$$
\Delta v_{i}=i_{\text {avg }} k_{i}
$$

where $k_{i}$ is the shift gain which is selected close to the value of $R_{d}$ so that the impact of voltage shift can be compensated.

\section{Proposed Control Technique}

A SMC technique to eliminate circulating currents based on distributed architecture is proposed in this paper. The inner voltage and current loop of the PECs are controlled through SMC as shown in Figure 3. It is a nonlinear controller which is used for variable structured systems and ensures stability in all operating conditions [10].

\section{Simulation Results and Discussion}

A two-source standalone DC microgrid sharing a common load is simulated for the parameters given in Table 1. To compare the results with the proposed method, a twosource DC microgrid is simulated with a droop control method and results are shown in Figure 4. For a small value of droop resistance $R_{d}=0.2 \Omega$, the observed output node voltages of sources 1 and 2 are 47.2 and $46.8 \mathrm{~V}$, and circulating currents $i_{c 1}$ and $i_{c 2}$ are -1 and $1 \mathrm{~A}$, respectively. These results show that circulating currents cannot be eliminated for a small value of droop resistance. For a large value of droop resistance $R_{d}=1.9 \Omega$, the circulated currents are -0.2 and $0.2 \mathrm{~A}$ but voltage deviation increases to $16 \%$, which is 
not acceptable by the loads. The same microgrid is simulated with distributed architecture using SMC and results are shown in Figure 5. In this case, the observed output node voltages of sources 1 and 2 are 47.8 and $46.8 \mathrm{~V}$, and circulating currents $i_{c 1}$ and $i_{c 2}$ are -0.05 and $0.05 \mathrm{~A}$, respectively. This confirms the performance of the proposed method. Moreover, to prove the reliability of the distributive architecture, a three-source DC microgrid sharing a common load is simulated for source failure condition and shown in Figure 6. At $0.1 \mathrm{~s}$, source 3 fails to supply current and the other two sources take up the load. This scenario shows the reliable performance of the distributed architecture.

Table 1. Parameters of DC microgrid.

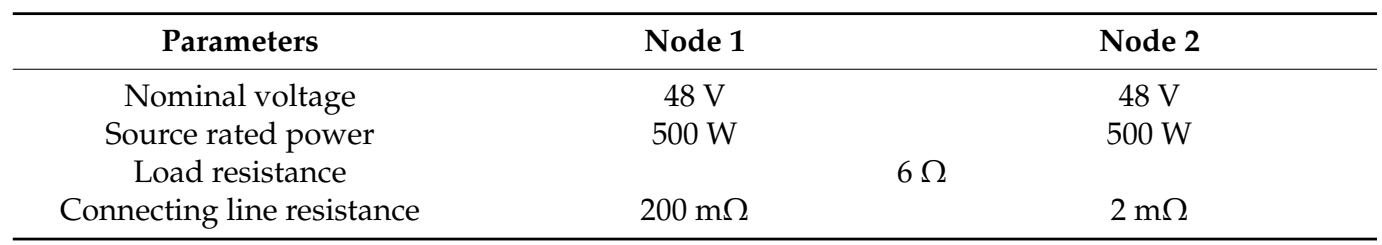
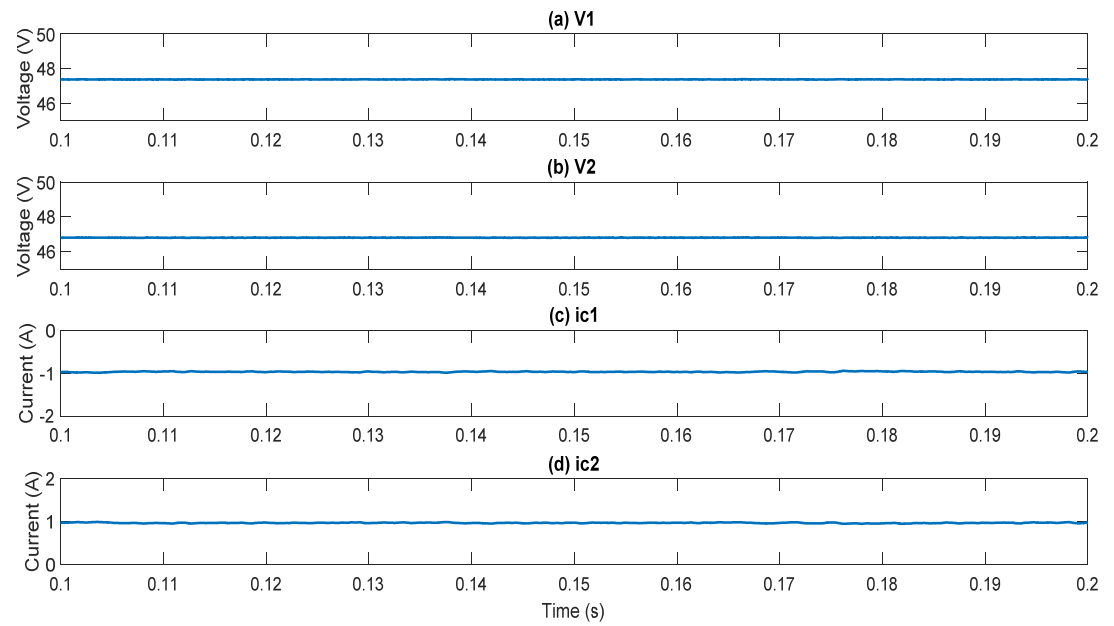

Figure 4. Circulating currents with droop control.
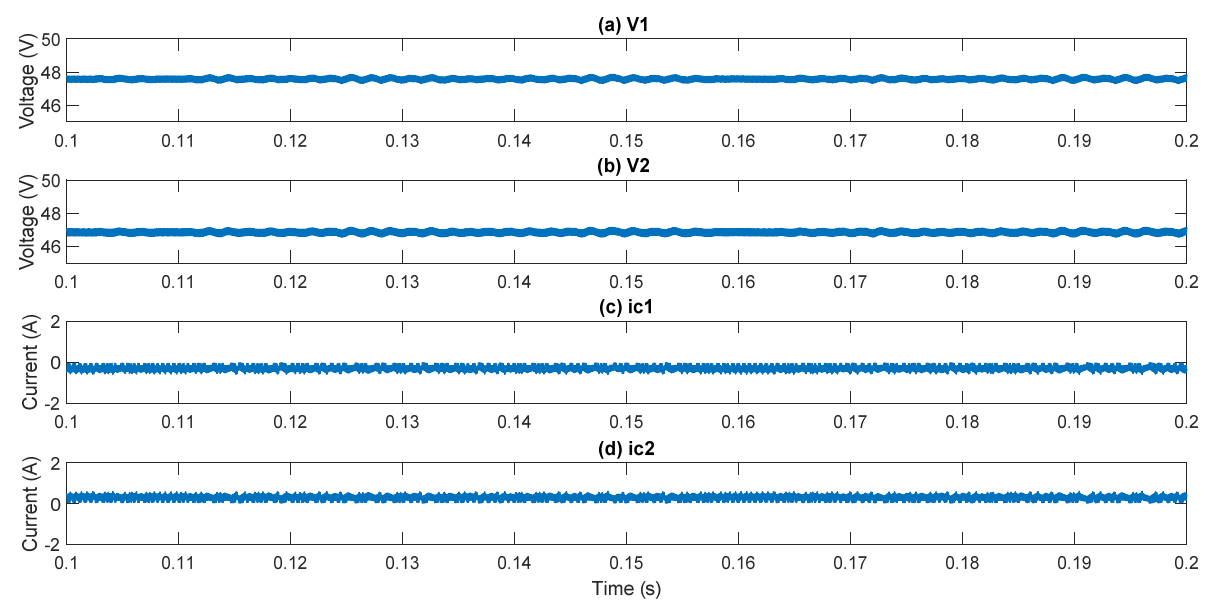

Figure 5. Circulating currents with SMC. 

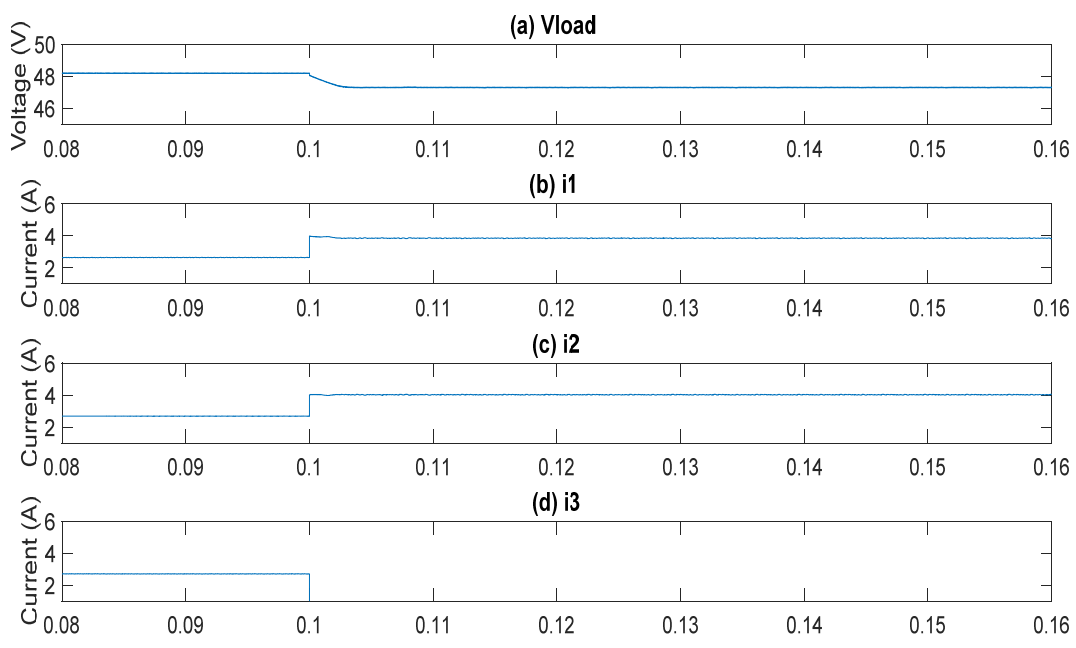

Figure 6. Fail-safe operation of the proposed control.

\section{Conclusions}

Sources in a standalone DC microgrid are interconnected in a parallel configuration. Among paralleled sources, there arises a major problem of circulating currents which disturb current sharing and voltage regulation. Droop controllers based on decentralized architecture are normally preferred for the mitigation of circulating currents. However, droop controllers cannot eliminate circulating currents and voltage deviation simultaneously. Solutions based on centralized control architecture can achieve the objective but single-point failure can degrade performance and reliability. To address the problem, a distributed architecture-based SMC technique is presented in this paper. Simulation results of steady-state conditions are presented to show the effectiveness and fail-safe operation of the proposed technique in a steady-state condition.

\section{References}

1. Salomonsson, D.; Sannino, A. Low-voltage DC distribution system for commercial power systems with sensitive electronic loads. IEEE Trans. Power Deliv. 2007, 22, 1620-1627. [CrossRef]

2. Kakigano, H.; Miura, Y.; Ise, T. Low-voltage bipolar-type DC microgrid for super high quality distribution. IEEE Trans. Power Electron. 2010, 25, 3066-3075. [CrossRef]

3. Carrasco, J.M.; Franquelo, L.G.; Bialasiewicz, J.T.; Galvan, E.; PortilloGuisado, R.C.; Prats, M.A.M.; Leon, J.I.; Moreno-Alfonso, N. Power-electronic systems for the grid integration of renewable energy sources: A survey. IEEE Trans. Ind. Electron. 2006, 53, 1002-1016. [CrossRef]

4. Guerrero, J.M.; Chandorkar, M.; Lee, T.L.; Loh, P.C. Advanced control architectures for intelligent microgrids 2014. Part I: Decentralized and hierarchical control. IEEE Trans. Ind. Electron. 2013, 60, 1254-1262. [CrossRef]

5. Yuan, M.; Fu, Y.; Mi, Y.; Li, Z.; Wang, C. Hierarchical control of DC microgrid with dynamical load power-sharing. Appl. Energy 2019, 239, 1-11. [CrossRef]

6. Nawaz, A.; Wu, J.; Long, C. Mitigation of circulating currents for proportional current sharing and voltage stability of isolated DC microgrid. Electr. Power Syst. Res. 2020, 180, 106123. [CrossRef]

7. Rashad, M.; Ashraf, M.; Bhatti, A.I.; Minhas, D.M. Mathematical Modeling and Stability Analysis of DC Microgrid Using SM Hysteresis Controller. Int. J. Electr. Power Energy Syst. 2018, 95, 507-522. [CrossRef]

8. Tayab, U.B.; Roslan, M.; Hwai, L.J.; Kashif, M. A review of droop control techniques for microgrid. Renew. Sustain. Energy Rev. 2017, 76, 717-727. [CrossRef]

9. Mahmoodi, M.; Gharehpetian, G.B.; Abedi, M.; Noroozian, R. Control systems for independent operation of parallel dg units in dc distribution systems. In Proceedings of the 2006 IEEE International Power and Energy Conference, Putra Jaya, Malaysia, 28-29 November 2006; pp. 220-224.

10. Utkin, V.; Guldner, J.; Shi, J. Sliding Mode Control in Electromechanical Systems; Taylor and Francis: London, UK, 1999. 